\title{
Antisense Oligonucleotide Therapies for Neurodegenerative Disorders
}

Blair R. Leavitt ${ }^{1}$ and Sarah J. Tabrizi ${ }^{2}$

1. Centre for Molecular Medicine \& Therapeutics, and Centre for Huntington's Disease at UBC Hospital, Department of Medical Genetics and Division of Neurology, Department of Medicine, University of British Columbia and BC Children's Hospital, Vancouver, BC, V5Z 4H4 Canada

2. Huntington's Disease Centre, Department of Neurodegenerative disease, and UK Dementia Research Institute at UCL, UCL Queen Square Institute of Neurology, University College London, WC1N 3BG, UK

Email - Sarah J. Tabrizi at s.tabrizi@ucl.ac.uk or Blair R. Leavitt at bleavitt@cmmt.ubc.ca

Summary sentence: Antisense oligonucleotide-based approaches in development for Huntington's disease and for other neurodegenerative diseases associated with aberrant protein production have enormous potential as disease-modifying therapies.

Antisense oligonucleotides (ASOs) have the potential to reduce, restore, or modify RNA and protein expression and thus can target disease pathogenesis by altering the expression of mutant proteins (1). The recent regulatory approval of ASObased therapy for the pediatric motor neuron disease spinal muscular atrophy has provided a regulatory pathway for additional ASO therapies in other central nervous system (CNS) diseases. Developments in ASO chemistry and advances in CNS delivery methods have enabled ASOs to enter clinical trials to treat Huntington's disease (HD) and other neurodegenerative disorders. There are currently no available treatments that slow or prevent progression of HD, but two ongoing ASO-based clinical programs have shown promising results to date. In 
addition, ASO-based clinical trials are currently underway in amyotrophic lateral sclerosis (ALS), Parkinson's disease, Alzheimer's disease, and are in development for other neurodegenerative diseases. It is hoped that ASO-based approaches will provide effective disease-modifying therapies for HD and similar neurodegenerative diseases in the near future.

ASOs are generally 16-22 bases long synthetic single-stranded DNA analogues that selectively bind to specific complementary RNA targets. A limitation of the original ASOs developed for clinical use was susceptibility to rapid degradation by cellular nucleases, but chemical modifications have since been introduced to improve their therapeutic utility. For example, substitution of sulphur for oxygen and modification of the 2'-position of the sugar to generate 2'-O-methoxyethyl (MOE)-modified ASOs with a phosphorothioate backbone resulted in improved nuclease resistance, potency and better tolerability in patients. Further modifications of the ribose sugar moiety have led to improved efficacy by improving binding to target RNAs (2).

ASOs can modulate target gene expression through numerous pathways (2). One such pathway is through ribonuclease (RNase) $\mathrm{H} 1$ recruitment (see the figure). Following selective binding of the ASO to its target RNA, an RNA-DNA hybrid is formed, which induces mRNA degradation by RNase $\mathrm{H} 1$. Other pathways depend on the specific location of ASO binding to target RNA (2). For example, ASOs can target intron-exon junctions in pre-mRNA to modulate RNA splicing. ASOmediated target suppression can be achieved by modulating splicing to introduce an out- of- frame deletion, which results in protein knockdown by nonsensemediated decay of the corresponding transcript. ASOs targeting translation start sites in RNA can block the binding of ribosomes, leading to complete translational inhibition of target protein synthesis.

Decreasing pathogenic protein expression is an approach that has potential benefit for many neurodegenerative diseases, such as lowering mutant huntingtin (mHTT) 
protein in HD, superoxide dismutase 1 in ALS caused by SOD1 mutations, or asynuclein in Parkinson's disease. ASOs have several advantages over RNA interference approaches in targeting disease-causing proteins. ASOs are readily taken up by neurons and have clear dose-dependent and reversible effects. ASOs also have the advantage that they will not saturate endogenous microRNA pathways, a potential cause of toxicity in short inhibitory RNA (siRNA)-based approaches. ASOs are generally highly selective and can target both introns and exons because they bind to pre-mRNA rather than mature mRNAs, allowing selection of unique target sequences for ASOs that do not appear anywhere else in the genome. However, unlike viral-mediated siRNA approaches, repeated administration of ASOs is required to maintain therapeutic effects. However, this may also be an advantage given that the effects of chronic suppression of target RNA (or perhaps an off target RNA) is not known prior to a human trial when suppression occurs for many years, ASOs have an advantage that if an unwanted outcome were to become apparent there is an off-switch (ie their effects are fully reversible) which is much less so the case for AAV-siRNAs.

$\mathrm{HD}$ is an inherited autosomal dominant neurodegenerative disorder characterized by a triad of motor, cognitive and psychiatric features. HD typically arises in midlife, with inexorable progression of disability over $10-15$ years. HD is caused by an abnormally expanded CAG repeat expansion in the HTT gene, which is expressed as a long polyglutamine tract in the mHTT protein that confers a toxic gain-offunction (3). Proposed pathological mechanisms caused by this alteration in HD include early transcriptional dysregulation, synaptic dysfunction, altered axonal vesicular trafficking, impairment of the proteostasis network, mHTT aggregate pathology, impairment of the nuclear pore complex and nuclear-cytoplasmic transport, oxidative damage, mitochondrial dysfunction and extra-synaptic excitotoxicity (3). ASOs provide a direct approach to reduce mHTT expression by targeting its RNA for destruction, thus preventing translation of $\mathrm{mHTT}$ and proximally targeting the primary disease mechanism (1). Two ASO-based therapeutic programs have recently entered clinical testing for the treatment of HD. 
The HTT-targeting ASO RG6042 acts via RNase H1 to target both wild-type and mutant HTT pre-mRNA and results in HTT-lowering. Preclinical studies of similar HTT-targeting ASOs in transgenic HD mouse models demonstrated decreased mHTT concentrations in brain tissue, correction of striatal gene transcriptional dysregulation, and phenotypic improvement (4). mHTT lowering was prolonged following even a single cerebral spinal fluid (CSF) injection of ASO in these mice suggesting that the effects of these ASOs will be prolonged in HD patients. Lumbar intra-thecal infusion of a similar ASO into non-human primates was also shown to effectively lower HTT in many brain regions relevant for HD pathology (4).

In the initial phase $1 / 2 \mathrm{a}$ trial of intra-thecal RG6042, treatment of 46 patients resulted in significant dose-dependent reduction in CSF concentrations of $\mathrm{mHTT}$ by $40-60 \%(5)$. The CSF concentrations of $\mathrm{mHTT}$ continued to decline during this short study, suggesting maximal reduction was not reached. The amount of CSF mHTT reduction observed in this study is consistent with the reductions in $\mathrm{mHTT}$ required for significant phenotypic improvement in transgenic mouse models of HD (4). Intra-thecal delivery of RG6042 ASO has been shown to be safe and well tolerated in HD patients, and its potential effects on disease modification and clinical outcomes is being assessed as part of GENERATION HD1, a large phase 3 trial involving over 800 early stage HD patients (NCT0376184).

Selective lowering of $\mathrm{mHTT}$ is theoretically a very attractive approach to therapy for HD and would overcome any concerns about the potential loss of wild-type HTT function caused by ASOs that target both HTT alleles. Selective mHTT lowering ASOs that target specific single nucleotide polymorphisms (SNPs) in linkage to the CAG expansion show promise in pre-clinical models of HD (6). A chemistry platform has been developed allowing the chirality of the phosphorothioate modification to be controlled during ASO synthesis. "Stereopure" ASO drugs generated with this platform have relatively selective targeting of $\mathrm{mHTT}$ over wildtype HTT in vitro. It is reported that "stereopure" ASOs have improved activity, 
stability, and specificity compared with stereoisomer mixtures (7). Two clinical trials were initiated in 2017 to assess two different "stereopure" ASOs targeting specific SNPs in linkage disequilibrium with the CAG expanded HTT allele. These studies require very precise genotyping to ensure the targeted SNP is accurately phased to the HTT allele with the CAG expansion. Not all individuals with HD can be treated with this SNP-based approach, and it is estimated that the two ASOs being tested will cover about $65 \%$ of HD patients from North America or Europe. Each ASO is given as a monthly intra-thecal bolus over three consecutive months, and it was recently reported that at the highest dose tested, one of the ASOs caused a modest $12.4 \%$ reduction of CSF mHTT concentration. An additional higher-dose cohort will now be added to this trial (NCT03225833).

It is not known which of the ASO approaches currently in clinical testing, HTTlowering or mHTT-selective lowering, is going to be most effective. Both approaches have distinct strengths and limitations, and this question will ultimately be answered empirically in clinical efficacy trials. Pre-clinical studies in a humanized transgenic HD mouse model of HD found that the benefits of ASOmediated lowering of total HTT levels (both $\mathrm{mHTT}$ and wtHTT) by $75 \%$ were similar to that of ASOs that selectively reduced $\mathrm{mHTT}$. These and other studies have suggested that the degree of $\mathrm{mHTT}$ lowering is the most critical parameter for preclinical efficacy in HD models (8). A substantial advantage of HTT lowering approaches compared to SNP-based selective mHTT targeting is the potential to develop a single therapeutic agent for the entire HD population. This work has led to considerable optimism that HTT-targeting ASOs may lead to a viable diseasemodifying therapy not just for HD, but also for the development of ASOs for other neurodegenerative diseases associated with aberrant protein production.

Following a substantial pre-clinical development program, an early stage ASO targeting SOD1 was evaluated in a phase 1 trial for the treatment of SOD1 mutation-positive ALS and shown to be safe and well tolerated following lumbar intra-thecal infusion (9). A more potent MOE-modified ASO (ISIS-SOD1Rx) is 
currently being evaluated in a phase $1 / 2$ a clinical trial (NCT02623699). Promising pre-clinical data have also been generated using ASO-based approaches for Parkinson's disease targeting leucine rich repeat kinase 2 (LRRK2), which is pathologically mutated (NCT03976349) and $\alpha$-synuclein, the pathologic protein in Lewy bodies of Parkinson's patients (10); Alzheimer's disease targeting Tau protein (11) which is currently in a phase 1 clinical trial (NCT03186989); and $\operatorname{Pr} P$ RNA for prion diseases (12).

ASO-based therapies are also of interest in diseases similar to HD such as the polyglutamine protein-related forms of spinocerebellar ataxia (13) and frontotemporal dementia (FTD) with Tau or TAR DNA-binding protein 43 (TDP-43) pathologic protein inclusions. Indeed, ASOs that lower ataxin2 levels have shown benefit in mouse models of both spinocerebellar ataxia 2 and TDP-43 related FTD (14). The most common genetic cause of ALS and FTD is a GGGGCC repeat expansion in the C9orf72 gene that causes disease by inducing RNA-mediated neurotoxicity. ASOs that selectively target these repeat-containing RNAs may be a useful therapeutic approach to this class of diseases (15). Clinical trials based on these promising pre-clinical results are in early development.

ASOs have already changed the landscape of therapeutic development for neurodegenerative diseases. The future advancement of ASOs in the clinic for CNS diseases will require continued development and research, including optimization of target sequence selection, improving biological activity, testing new delivery technology, and maintaining an appropriate safety profile. Improving the delivery of ASO to target cells is an important area of future development including novel forms of ASO delivery to the CNS such as intra-thecal pumps and the use of lipid-based and polymer-based nanocarriers. Such delivery systems will potentially improve the controlled release of ASOs, be more cell and tissue specific, and may provide additional protection for the ASOs from nuclease degradation. Beyond potency and specificity, another crucial feature of a good candidate molecule is the ability to reach its intracellular target at sufficient 
concentration. Given the substantially increased potency of MOE-modified ASOs, safety concerns regarding both excessive on-target lowering of target proteins (such as wild-type HTT in HD) and potential off-target effects exist, and it is critical that extensive pre-clinical assessment of both potency and off-target effects is performed in the development of new ASO therapies.

The ASO RG6042 now in phase 3 clinical trials is the result of more than a decade of extensive pre-clinical assessment in multiple model systems and stands as a testament to effective academic and industry collaboration in drug development. With a growing number of ASO-mediated therapeutics now being tested in clinical trials, this exciting technology holds the potential to change the therapeutic landscape for many neurological and non-neurological conditions (including cancer, cardiovascular, infectious and pulmonary diseases) in the near future. 


\section{REFERENCES AND NOTES}

1. S.J. Tabrizi SJ, R. Ghosh R, B.R. Leavitt, Neuron 102, 899 (2019).

2. C. Rinaldi, M.J.A. Wood, Nat. Rev. Neurol. 14, 9 (2018).

3. G.P. Bates et al., Nat. Rev. Dis. Prim. 1, 15005 (2015).

4. H.B. Kordasiewicz et al., Neuron 74, 1031 (2012).

5. S.J. Tabrizi SJ, et al., N Eng. J. Med. 380, 2307 (2019).

6. N.H. Skotte et al., PLoS One, 9, (2014)

7. N. Iwamoto et al., Nat. Biotech. 35, 845 (2017).

8. A.L. Southwell et al., Sci Transl Med, 10. (2018).

9. T.M. Miller et al., Lancet Neurology 12, 435 (2013).

10. H.T. Zhao et al., Mol. Ther. Nucleic Acids 8, 508 (2017).

11. S.L. DeVos et al., J. Neuroscience 33, 12887 (2013).

12. G.J. Raymond et al., JCl Insight 4, 131175 (2019).

13. P. Gonzalez-Alegre Hum. Mol. Genet. 28, R80 (2019).

14. L.A. Becker et al., Nature 544, 367 (2017).

15. J. Jiang et al., Neuron. 90, 535 (2016).

\section{ACKNOWLEDGMENTS (OPTIONAL)}

Both authors have undertaken paid consultancy work for lonis pharmaceuticals, Takeda pharmaceuticals, and F Hoffman-La Roche. 